\title{
Do the benefits of folic acid fortification outweigh the risk of masking vitamin $B_{12}$ deficiency?
}

Due to an editing error in the No argument by Edward Reynolds in this Head to Head (BMJ 2018;360:k724, doi:10.1136/bmj. $\mathrm{k} 724$ ), we incorrectly stated that total homocysteine and methylmalonic acid concentrations decreased (not increased) with increasing folate in participants with low serum vitamin B12 levels. 